\title{
Phonons in mesoporous silicon: the influence of nanostructuring on the dispersion in the Debye regime
}

\author{
Tommy Hofmann ${ }^{\mathrm{a}}$, Dirk Wallacher ${ }^{\mathrm{a}}$, Rasmus Toft-Petersen ${ }^{\mathrm{a}}$, Britta Ryll ${ }^{\mathrm{a}}$, \\ Manfred Reehuis ${ }^{\mathrm{a}}$, Klaus Habicht ${ }^{\mathrm{a}}$ \\ ${ }^{a}$ Helmholtz-Zentrum Berlin für Materialien und Energie GmbH, 14109 Berlin, Germany
}

\begin{abstract}
We present a comprehensive scattering study of nanostructured silicon. Neutron and x-ray scattering experiments elucidate structural and dynamical properties of electrochemically etched, porous silicon membranes with pores roughly $8 \mathrm{~nm}$ across. In particular, inelastic cold neutron scattering techniques reveal the phonon dispersion of the nanostructured, single crystalline samples in the linear Debye regime for energy transfers up to $4 \mathrm{meV}$. A modified dispersion relation characterized by systematically reduced sound velocities manifests itself in altered elastic properties of porous silicon when compared to bulk silicon. Its relevance for nanostructured silicon as thermoelectric material of interest is discussed.
\end{abstract}

Keywords: mesoporous silicon, inelastic neutron scattering, phonon dispersion, elasticity, thermoelectric material

\section{Introduction}

Thermoelectric materials convert temperature gradients into electricity and vice versa electric currents into cooling power [17]. Consequently, thermoelectric solid state devices are expected to play a key role in the global search for sustainable and green energy technologies. The efficiency of state-of-the-art materials however is limited. This efficiency directly relates to the figure of merit $Z T=\sigma S^{2} \kappa^{-1} T$, which depends on electrical conductivity $\sigma$, Seebeck coefficient $S$, temperature $T$, and thermal conductivity $\kappa$ and strategies must be devised to tailor the performance of materials by tuning electrical and thermal conductivity independently to increase $Z T$.

Skutterudites [53, 38], half- and full-Heusler alloys [21, 60, 46, 56] and more generally nano- and hierarchically-structured materials $[20,58,44]$ are among the most discussed and studied systems to exhibit superior thermoelectric performances [52]. Key strategies to manipulate $Z T$ are introducing local disorder in the crystal structure in form of rattler atoms, defining scattering centers on

URL: tommy.hofmann@helmholtz-berlin.de (Tommy Hofmann) 
various length scales for phonons and phonon dispersion engineering through nanostructuring.

Studying novel thermoelectric materials commonly involves more or less sophisticated synthesis procedures, macroscopic characterization of thermal and electronic transport and structural investigations by means of scattering techniques. Less frequent are studies of the microscopic origin of heat transport [11] to relate thermal conductivity to peculiarities of phonon dispersion or diffuse phonon scattering. Similarly rare are synchrotron based spectroscopic studies [41] to elucidate the electronic properties in terms of band structure or isolated electronic levels, which define the power factor $P F=\sigma S^{2}$.

A multitude of studies discuss or propose nanostructured silicon as thermoelectric material of interest $[37,7,19,9,55,48,13,11,49,54]$. In particular, it is envisioned to manipulate thermal conductivity by isotopic disorder [45], to increase diffuse scattering of phonons at artificial interfaces, e.g., pore walls $[48,49]$ and to manipulate the phonon dispersion relation itself for instance via band gap engineering [27]. Whereas theoretical studies on this subject appear numerous, there exist only a few experimental investigations [13, 55, 39] and rarely ones, which go beyond macroscopic sample characterization [11].

There are different routes to structure silicon on nanometer sized length scales. E-beam lithography is one of the most sophisticated approaches. It provides the greatest flexibility in terms of defining regular nanometer-sized surface patterns but comes at the cost of demanding technological requirements, a small sample throughput and financially challenging expenditures [24]. Finally, the technique is limited to the synthesis of only several nanometer thick structured films. Electrochemical etching of silicon provides a low-tech approach to define nanometer features [59]. It offers the possibility to synthesize macroscopic amounts of porous, single crystalline silicon at low costs and on feasible time scales although with reduced structural regularity. The single crystalline nature of such samples renders them particularly interesting for structural and dynamical studies utilizing scattering techniques. Nanowire growth [47, 43], template assisted reactive ion etching [55] and nanoparticle synthesis [25] are other regularly employed synthesis methods with different degrees of complexity.

Here, we present elastic and inelastic neutron scattering experiments to probe structural and dynamical properties of porous silicon membranes ( $\mathrm{pSi}$ ) as a model system. In particular, inelastic neutron scattering experiments utilizing cold neutrons emerge as valuable tool to elucidate modified dispersion relations in nanostructured silicon samples in the Debye regime with the potential to be extended towards the Brillouin zone boundary with thermal neutrons or x-rays. These methods are of immense interest in the field of phonon engineering and its expected impact in the field of thermoelectrics [3, 45, 31].

Subsequent to this introductory part, the remainder of our article is organized as follows. Chapter II and III discuss sample preparation and characterization. Section IV provides details on the inelastic neutron scattering experiments performed on porous silicon samples. Section $V$ recalls some basics of elasticity theory, whereas one can find details of the data analysis in chapter VI. Chapter VII finally provides a detailed discussion of experimental results. As a highlight 
we present modified elastic properties of nanostructured silicon as evident from our studies.

\section{Experiment}

\subsection{Samples}

The term porous silicon refers commonly to electrochemically etched silicon, which exhibits micro- or nanometer-sized cavities in an otherwise single crystalline matrix $[10,50,59]$. Its synthesis employs usually hydrofluoric acid (HF) as electrolyte and its morphology is defined in a fairly convoluted way by the etching parameters as HF concentration and applied current as well as doping and orientation of the source material that are single crystalline Si wafers [59]. Adjusting these parameters allows tailoring pore diameters, porosities and morphologies ranging from complex, interconnected pore networks to tubular, aligned channels several hundred microns long.

The synthesis of our samples encompassed three steps: electrochemical etching, epilayer separation and crystallographic cutting. To grow roughly $8 \mathrm{~nm}$ wide tubular channels along the [001] direction, $p$-doped [001] silicon wafers with a resistivity of $\rho=0.01-0.02 \Omega \mathrm{cm}^{-1}$ were etched in an hydrofluoric acid/ethanol solution $[\mathrm{HF}(48 \%): \operatorname{Eth}(99.9 \%)=4: 6]$ for 4 h applying a current density of $j$ $=18 \mathrm{~mA} \mathrm{~cm}^{-2}$. This etching process was terminated by increasing the current density to $j=215 \mathrm{~mA} \mathrm{~cm}^{-2}$ for 40 s to detach the porous epilayer of roughly $160 \mu \mathrm{m}$ thickness from the supporting silicon wafer creating free standing pSi membranes. Subsequently, the cleaned and dried membranes were cut with a laser into quadratic, $30 \times 30 \mathrm{~mm}^{2}$ membranes along the crystallographic [110] and $[1 \overline{1} 0]$ directions.

In total, 38 membranes were synthesized and assembled to form a $6 \mathrm{~mm}$ stack of pSi providing sufficient material for neutron scattering experiments. The surface normal of the stack coincided with the [001]-direction and the edges were parallel to [110] and [1 $\overline{1} 0]$. An equivalent stack of bulk silicon (bSi) plates was prepared from the same batch of Si wafers and served as reference sample to calibrate instrumental offsets utilizing its well known structural and dynamical properties.

\subsection{Characterization}

Nitrogen sorption isotherms are a standard metrology to ascertain morphological characteristics of mesoporous materials $[10,50]$. At fixed sample temperature $T=77 \mathrm{~K}$, volumetric measurements relate the liquid nitrogen uptake $f=N / N_{0}$ in mesopores to the vapor pressure $P_{\text {red }}=P / P_{0}$ of the coexisting vapor phase, where $N$ labels the number of physisorbed $\mathrm{N}_{2}$ molecules, $N_{0}$ the number of molecules required to fill the entire pore space, $P$ the vapor pressure and $P_{0}$ the equilibrium saturation pressure of bulk nitrogen at $77 \mathrm{~K}$.

A nitrogen sorption isotherm typically follows an hysteretic path upon adsorption and desorption, which reflects layer growth on the pore walls at small reduced pressures $P_{\text {red }}<P_{\text {red }}^{a d / d e}$ and capillary condensation that is the formation 
of liquid drops in the pore center above $P_{\text {red }}=P_{\text {red }}^{a d / d e}$. This peculiar behavior allows inferring effective surface area $A$, pore size distribution and porosity $\Phi$ of the substrate by BET-analysis [8], BJH-analysis [6] or more sophisticated DFT-calculations [35].
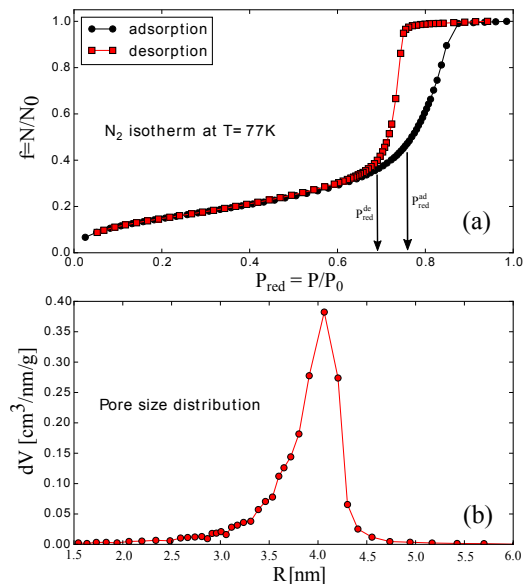

Figure 1: (Color online) (a) $\mathrm{N}_{2}$ sorption isotherm measured at $T=77 \mathrm{~K}$ in pSi. (b) Pore size distribution in $\mathrm{pSi}$ estimated from the desorption branch of the $\mathrm{N}_{2}$ isotherm employing a BJH-analysis.

Fig. 1(a) exhibits a representative sorption isotherm of the synthesized pSi samples. A BET-analysis of the initial part of the isotherm $\left(P_{\text {red }}<0.3\right)$ predicts an effective surface of $A=157 \mathrm{~m}^{2} \mathrm{~g}^{-1}$. A BJH-analysis relates the desorption pressures upon capillary condensation via the thickness of the coexisting wall film $t\left(P_{\text {red }}\right)$ and the Kelvin equation $k_{B} T \ln \left(P_{\text {red }}\right) \sim\left[R-t\left(P_{\text {red }}\right)\right]^{-1}$ to the pore size distribution in Fig. 1(b) with an average radius of $R \approx 4 \mathrm{~nm}$ and cut-offs close to $2.5 \mathrm{~nm}$ and $5.5 \mathrm{~nm}$. Membrane volume and $N_{0}$ allow a porosity estimate of $\Phi \approx 50 \%$.

Scanning electron microscopy (SEM) provides a more direct approach to visualize the morphology of pSi membranes. It presents naturally a more local, surface sensitive characterization of the samples rather than a volume sensitive probe like a sorption isotherm. A SEM image of the top view of a pSi membrane is shown in Fig. 2(a). It displays the polygonal shape of the pores as reported earlier [12]. The inset shows the size distribution of pores at the membrane surface. This distribution has been obtained by a contour finding algorithm [40], applied to a binary version of the 2D-SEM image and subsequently relating the enclosed areas of polygonal-shaped contours to radii of circles with equivalent area. Fig. 2(b) exhibits the tree-like morphology of the pore-channels along the [001] direction caused by dendritic growth during the etching process [59].

In order to probe the quality of the alignment of the individual membranes in the assembled stack, diffraction experiments were performed on the $\gamma$-ray diffractometer at the HZB, which is equipped with a ${ }^{192}$ Ir source giving a wavelength of $\lambda=0.0392 \AA$ (Ref. [28]). In $\omega$-scans at fixed scattering angles for 
selected Bragg reflections (111, 220 and 004) the samples were rotated and the intensity variations as function of sample orientation $\omega$ recorded. From these measurements effective, accumulated mosaicities due to stacking multiple membranes of less than $\pm 0.5^{\circ}$ half width maximum were inferred. Consequently, the prepared sample stacks were considered to be perfectly suited for the planned neutron scattering experiments.
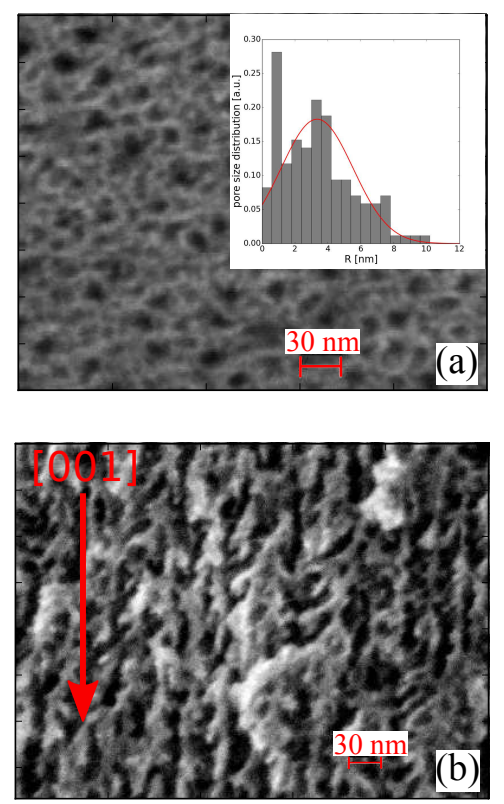

Figure 2: (Color online) SEM micrograph of pSi recorded with a SEM LEO GEMENI microscope employing electron energies of $15 \mathrm{keV}$ : (a) Top view of the pores in etched silicon. The inset illustrates the pore size distribution as inferred from a contrast analysis of the SEM image. (b) Side view of the pores.

\subsection{Neutron scattering}

The cold neutron triple-axis spectrometer FLEXX [36] at the research reactor BER II in Berlin was used for scattering studies. Corresponding sets of elastic and inelastic scattering experiments were performed on $\mathrm{pSi}$ and $\mathrm{bSi}$ to achieve a thorough understanding of the influence of nanostructuring on crystal structure and phonon dispersion. In all the experiments the [110] crystallographic plane of silicon as defined by the [001]- and [110] directions coincided with the scattering plane. For the measurements, samples were kept at room temperature in an evacuated and vacuum tight aluminum container.

Elastic scattering experiments employed neutrons with wavelengths $\lambda=2.24$ and $4.83 \AA$ to measure the wave vector dependent intensity of 004-, 220-, and 111-Bragg reflections longitudinally along the [001]-, [110]-, and [111]directions (Fig. 3). For pSi, these measurements are sensitive to structural 
anisotropies, because wave vector transfers $\mathbf{Q}$ parallel and perpendicular to the tubular nanochannels along [001] are probed. Phonons were studied at 111-
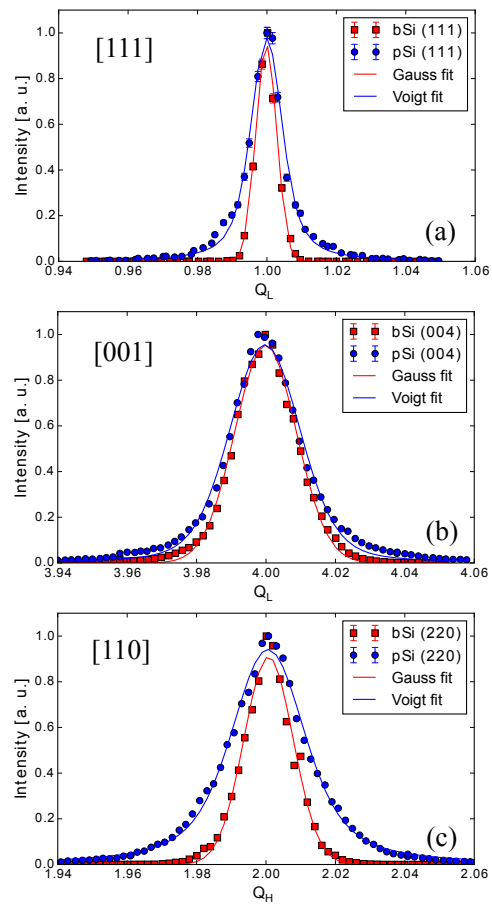

Figure 3: (Color online) Normalized elastic scattering intensity of pSi (blue symbols) and bSi (red symbols) at selected Bragg reflections $H K L$ as scanned longitudinally along the $[H K L]$ directions: (a) Scan along the [111]-direction at the 111-Bragg reflection. (b) Scan along the [001]-direction at the 004-Bragg reflection. (c) Scan along the [110]-direction at the 220-Bragg reflection (bottom). Solid lines represent Voigt curves obtained by nonlinear least squares fitting of the data. $Q_{L}$ and $Q_{H}$ are $H$ - and $L$ - components of the wave vector transfer $\mathbf{Q}$ reduced to the size of a cubic unit cell $2 \pi / a$ in reciprocal space. Statistical error bars are usually of the symbol size or even smaller and therefore often indistinguishable from the symbols.

, 220- and 004-Bragg reflections in [111]-, [1,1,1]-, [001]- and [110]-directions to discern properly between longitudinal and transverse excitations. Constant energy scans at $2 \mathrm{meV}, 3 \mathrm{meV}$ and $4 \mathrm{meV}$ energy transfer $\Delta E$ allowed to determine the wave vector $\mathbf{q}$ of the phonons within the respective Brillouin zones. Fig. 4 exhibits such an exemplary, longitudinal [111] phonon as measured at the 111-Bragg reflection for $\Delta E=3 \mathrm{meV}$.

\subsection{Data analysis}

\subsubsection{Structure}

The elastic scattering data as shown in Fig. 3 were analyzed with respect to width and wave vector of the Bragg reflections. In particular, nonlinear least squares fitting of Voigt functions was employed to determine the full width 


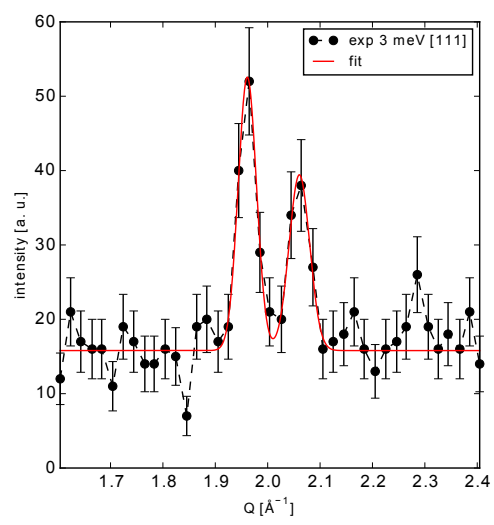

Figure 4: (Color online) Monolithic pSi sample: Longitudinal [111] phonon as measured at the 111 reflection for an energy transfer of $3 \mathrm{meV}$. The black line shows a nonlinear least squares fit of two Gaussians to the data to find the phonon wave vector $\mathbf{q}=\mathbf{Q}_{\max }-\mathbf{Q}_{\mathbf{B r a g g}}$ in the Brillouin zone.

half maximum (FWHM) of Gauss $\left(\gamma_{G}\right)$ and Lorentz $\left(\gamma_{L}\right)$ contributions to the observed peak profiles. Results are listed in Table 1.

\subsubsection{Phonon dispersion}

Inelastic scattering data as shown in Fig. 4 provide as main information the relation between wave vector $q$ and energy $\hbar \omega$ of the probed phonons $\omega(q)$. The determination of the phonon wave vector through nonlinear least squares fitting of Gaussian profiles to the constant-energy scans for different energies and crystallographic directions allowed obtaining the phonon dispersion in the linear Debye regime (Fig. 5) for longitudinal and transverse polarizations. The slope $v$ of the dispersion $\omega=v q$ in the Debye regimes represents the group velocity of corresponding sound waves and was estimated for pSi by linear least squares fitting.

Table 2 lists accepted literature values for the sound velocities of bSi. Consequently, in our data analysis the measured phonons for bSi were not used to ascertain the slopes of the phonon dispersion curves. Rather, linear least squares fitting assuming literature values for $v_{\text {sound }}$, was used to calibrate and correct the phonon wave vectors for an evident, q-independent instrumental offset close to the center of the Brillouin zone $\left(q \rightarrow 0 \AA^{-1}\right)$. The offset for $[111]_{L}$, for instance was $0.006 \AA^{-1}$. That is roughly $0.5 \%$ of the size of the entire Brillouin zone $2 \pi / a$.

\subsubsection{Elasticity}

In elasticity theory the rank-2 tensors strain $\sigma$ and stress $\epsilon$ are related via the inverse rank- 4 tensors of stiffness $C$ and compliance $S$. Generally, the relationship

$$
\sigma_{i j}=c_{i j k l} \epsilon_{k l} \quad \epsilon_{i j}=s_{i j k l} \sigma_{k l}
$$


with $i, j, k, l \in\{x, y, z\}$ holds for any crystalline structure. In cubic systems like silicon, however, symmetry considerations reduce the 81 entries of $S$ and $C$ to only three independent components. They are often referred to as $s_{11}, s_{12}$, and $s_{44}$ and $c_{11}, c_{12}$, and $c_{44}$, respectively (Ref. [26]).

In the long wavelength limit (Debye regime) the group velocity $v_{L / T}^{[H K L]}$ of sound waves in cubic crystals relates to the three independent components of the stiffness tensor and the mass density $\rho$. For longitudinal and transverse waves along [001], [110], and [111] directions it is

$$
\begin{aligned}
v_{L}^{[100]} & =\left(\frac{c_{11}}{\rho}\right)^{1 / 2} \\
v_{T}^{[100]} & =\left(\frac{c_{44}}{\rho}\right)^{1 / 2} \\
v_{L}^{[110]} & =\left(\frac{c_{11}+c_{12}+2 c_{44}}{2 \rho}\right)^{1 / 2} \\
v_{T, \|}^{[110]} & =\left(\frac{c_{44}}{\rho}\right)^{1 / 2} \\
v_{L}^{[111]} & =\left(\frac{c_{11}+2 c_{12}+4 c_{44}}{3 \rho}\right)^{1 / 2} \\
v_{T}^{[111]} & =\left(\frac{c_{11}-c_{12}+c_{44}}{3 \rho}\right)^{1 / 2} .
\end{aligned}
$$

Only for [110]-phonons the two existing transverse modes are degenerate. Therefore, in the fourth equation the symbol $\|$ explicitly refers to a polarization vector that resides in the scattering plane. We omitted the equation for the case of orthogonal polarization $v_{T, \perp}^{[110]}$, since respective phonons cannot be probed in our scattering geometry.

Uniaxial compression or elongation in one of the main crystallographic directions is in cubic systems expressed in terms of Young moduli $Y_{H K L}$ as defined by the compliance tensor [26]:

$$
\begin{aligned}
& \frac{1}{Y_{[100]}}=s_{11} \\
& \frac{1}{Y_{[110]}}=s_{11}-\frac{1}{2}\left[\left(s_{11}-s_{12}\right)-\frac{1}{2} s_{44}\right] \\
& \frac{1}{Y_{[111]}}=s_{11}-\frac{2}{3}\left[\left(s_{11}-s_{12}\right)-\frac{1}{2} s_{44}\right] .
\end{aligned}
$$

Additionally, in the silicon structure with the tetrahedral coordination of $\mathrm{Si}$ atoms the Keating relation [30] holds between the three components of the stiffness tensor leaving only two independent components:

$$
\Psi=\frac{2\left(c_{11}+c_{12}\right) c_{44}}{\left(c_{11}-c_{12}\right)\left(c_{11}+3 c_{12}\right)} \equiv 1
$$

Sound velocities were converted into elastic constants of pSi employing the set of 
equations above (Eqns 2). To find an adequate agreement between theoretical sound velocities (Eqns 2) and experimental values in Table II, we minimized the expression (5) with respect to the elastic constants $c_{11}, c_{12}$ and $c_{44}$. Here, the sum was taken over the probed crystallographic directions ([111], [110], [001]) and polarizations $\mathrm{p}(\mathrm{L}, \mathrm{T})$.

$$
\sum_{p, \text { crys.dir. }}\left(1-\frac{v_{p}^{\text {crys.dir. }}\left(c_{11}, c_{12}, c_{44}\right)}{v_{p, \text { exp }}^{\text {crys.dir. }}}\right)^{2} .
$$

In a next step, the inverse of the stiffness tensor $\mathrm{C}$ provided the entries of the compliance tensor, which finally defined the Young moduli via Eqns 3. The resulting elastic constants and uncertainties are listed in Table III.

Table 1: FWHM of Gauss and Lorentz contributions to the Voigt shaped Bragg reflections.

\begin{tabular}{|c|c|c|c|}
\hline & 111 & 220 & 004 \\
\hline \hline$\gamma_{G}^{b S i}\left[\AA^{-1}\right]$ & $0.0140(1)$ & $0.0280(2.4)$ & $0.0240(1.3)$ \\
\hline$\gamma_{L}^{b S i}\left[\AA^{-1}\right]$ & 0 & 0 & 0 \\
\hline$\gamma_{G}^{p S i}\left[\AA^{-1}\right]$ & $0.0140(1)$ & $0.0280(2.4)$ & $0.0240(1.3)$ \\
\hline$\gamma_{L}^{p S i}\left[\AA^{-1}\right]$ & $0.0120(2.2)$ & $0.0230(3.2)$ & $0.0070(2.6)$ \\
\hline
\end{tabular}

In this particular analysis scheme care appears to be in order with respect to successive error propagation and provided error bars. The fitting of phonon wave vectors $q$ accounts for statistical uncertainties in the measured scattering intensities $I$, which scale with $\sqrt{I}$. The associated covariance matrix is analyzed to obtain $\Delta q$. Similarly, the linear least squares approximation of the dispersion relation, predicts sound velocities $v$ and uncertainties $\Delta v$. They include errors in phonon wave vectors $\Delta q$. Finally, error bars given for stiffness and compliance tensor as well as Young moduli are the result of a statistical bootstrap method. They rely on the repetitive calculation of sought quantities using starting parameters that vary randomly but normal distributed around their mean values [14].

\section{Results and discussion}

The reciprocal lattice vector $\mathbf{Q}$ of the highest indexed Bragg reflection 004 translates for bSi into a lattice constant of $a=5.4273(2) \AA$ in favorable agreement with the literature value of $5.43053(7) \AA[42]$. For pSi one obtains $a=$ $5.4357(2) \AA$. That is a relative increase in $a$ by not more than $0.15 \%$ and in accordance with an earlier high resolution x-ray scattering study [5]. Theoretically, the slightly increased lattice constant should also manifest itself in a decrease of mass density by $0.5 \%$. Assuming unaltered elastic constants in pSi, this would lead to an insignificant increase in sound velocities in pSi (Eqn 2). This is in obvious contrast to our experimental results below and we utilize confidently the mass density $\rho=2.33 \mathrm{~g} \mathrm{~cm}^{-3}$ for bSi as well as pSi in the subsequent discussion. 
The peak shape analysis in Fig. 3 and Table 1 utilized Gauss and Voigt functions. It clearly indicated a pure Gaussian shape for bSi as expected for Bragg reflections of single crystals measured on a triple-axis spectrometer [51]. The full width half maxima (FWHM) of the bulk Bragg reflections illustrate the Q- and wave length dependent instrumental resolution. Assuming Gauss contributions as inferred from bulk reflections, pSi samples exhibited additionally significant Lorentz contributions to Voigt-shaped peaks. Performing a single reflection analysis, one relates the FWHM of these Lorentz contributions $\gamma_{L}$ to the crystallite size $D$ via the equation $\gamma_{L}=4 D^{-1}$ [4]. For the [001], [110] and [111] directions one infers crystallite sizes of $58 \mathrm{~nm}, 17 \mathrm{~nm}$ and $32 \mathrm{~nm}$ that are significantly larger than the pore-pore distance of $11 \mathrm{~nm}$, which is expected for cylindrical pores with Radius $R=4 \mathrm{~nm}$ and a porosity of $\Phi=50 \%$ on a hexagonal lattice. As also reported by Barla et al. [5] the coherence of the lattice obviously prevails in the pSi samples beyond the next nearest neighbor pore-pore distances. The largest crystallite size is found along [001] and is likely a consequence of preferential etching, which allows for larger coherent domains along this direction to endure the sample synthesis. This morphological anisotropy is also clearly indicated in the SEM-images (see Fig. 2). Earlier such anisotropies were related to textural growth of confined solids, e.g., alkanes in pSi [23].

The performed profile analysis does not independently determine the Gaussian contributions $\gamma_{G}^{p S i}$ for pSi reflections. Rather, it uses the resolution limited bulk values. Deviating, from this analysis scheme and fitting $\gamma_{G}^{p S i}$ yields no reliable/systematic differences to respective bulk values. As a consequence, an interpretation of the Gauss widths in terms of strain [4] becomes impossible for pSi.

Table 2: Sound velocities: The table provides longitudinal and transverse sound velocities of bSi (literature) and pSi (experiment). The last row lists the exact sound velocities expected for the experimental estimate of elastic constants as listed in Table III.

\begin{tabular}{|c|c|c|c|c|c|c|}
\hline & $\begin{array}{c}{[111]_{L}} \\
\mathrm{~m} / \mathrm{s}\end{array}$ & $\begin{array}{c}{[111]_{T}} \\
\mathrm{~m} / \mathrm{s}\end{array}$ & $\begin{array}{c}{[100]_{L}} \\
\mathrm{~m} / \mathrm{s}\end{array}$ & $\begin{array}{c}{[100]_{T}} \\
\mathrm{~m} / \mathrm{s}\end{array}$ & $\begin{array}{c}{[011]_{L}} \\
\mathrm{~m} / \mathrm{s}\end{array}$ & $\begin{array}{c}{[011]_{T}} \\
\mathrm{~m} / \mathrm{s}\end{array}$ \\
\hline \hline bSi (Ref. [26], Eqns 2) & 9356 & 5094 & 8433 & 5844 & 9134 & 5844 \\
\hline pSi (exp) & $8113 \pm 247$ & $4707 \pm 100$ & $7230 \pm 110$ & $5210 \pm 100$ & $8971 \pm 600$ & $4912 \pm 60$ \\
\hline pSi (Eqns 2, Table III) & 8287 & 4586 & 7584 & 5158 & 8116 & 5158 \\
\hline
\end{tabular}

The analysis of the inelastic neutron scattering experiments in Fig. 5 indicated sound velocities in pSi that are reduced by more than $10 \%$ when compared to their respective bulk values. By means of Eqns 2, one determines elastic constants (Table III) and a significant reduction of the stiffness tensor components by more than $20 \%$ becomes evident for nanostructured pSi with $R \approx 4 \mathrm{~nm}$ and $\Phi=50 \%$. It is important to state, that our scattering experiments probed phonons with wavelengths between roughly $4 \mathrm{~nm}$ and $20 \mathrm{~nm}$. Consequently, reported sound velocities and elastic constants cannot be interpreted in terms of an effective medium approach, which relates them only to the amount of missing material as expressed by the porosity $\Phi$. Rather a more local, microscopic characterization of the elastic properties is achieved. Given the porosity of $\Phi=50 \%$ 


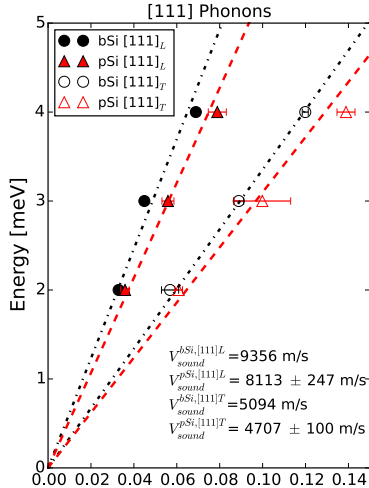
$\mathrm{q}\left[\AA^{-1}\right]$

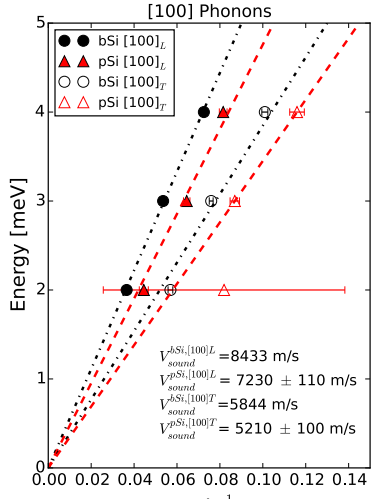

$\mathrm{q}\left[\AA^{-1}\right]$

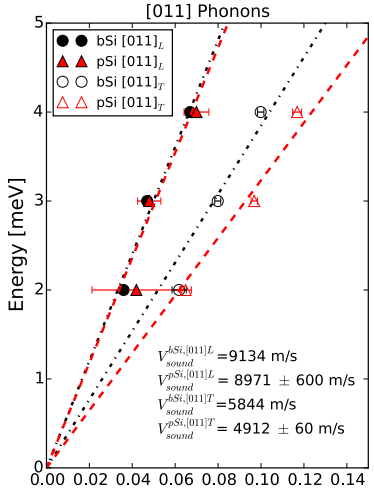

$\mathrm{q}\left[\AA^{-1}\right]$

Figure 5: (Color online) Phonon dispersion of pSi and bSi in the linear Debye regime. From left to right, symbols represent $[111]_{L / T},[100]_{L / T}$, and $[011]_{L / T}$ phonons. Dashed lines illustrate the linear dispersion relation close to the center of the Brillouin zone. For bSi the slopes of the dispersions translate exactly into the literature values for respective sound velocities (see text). Bulk data were used to reliably calibrate the instrumental offset close to the Brillouin zone center (see text). The slopes of the dispersions for the nanostructured sample were obtained by linear least squares fitting and provide the sound velocities in pSi. Shown data are corrected for the instrumental offset.

and the average pore size of $8 \mathrm{~nm}$, measured phonons elucidate strongly pore wall properties and less composite averages. Reported elastic moduli represent at least upper bounds for the modification of microscopic, elastic properties in pSi upon nanostructuring, which might be further refined in future scattering studies that probe the entire Brillouin zone in combination with atomistic simulations.

Table 3: Literature values for elastic constants of bSi and experimental values of $\mathrm{pSi}$ as inferred from measured sound velocities (Fig. 5), Eqns 2, and Eqn. 5.

\begin{tabular}{|c|c|c|c|c|c|c|c|c|c|}
\hline & $\begin{array}{c}c_{11} \\
\mathrm{GPa}\end{array}$ & $\begin{array}{c}c_{12} \\
\mathrm{GPa}\end{array}$ & $\begin{array}{c}c_{44} \\
\mathrm{GPa}\end{array}$ & $\begin{array}{c}s_{11} \\
10^{-3} \mathrm{GPa}^{-1}\end{array}$ & $\begin{array}{c}s_{12} \\
10^{-3} \mathrm{GPa}^{-1}\end{array}$ & $\begin{array}{c}s_{44} \\
10^{-3} \mathrm{GPa}^{-1}\end{array}$ & $\begin{array}{c}Y_{[100]} \\
\mathrm{GPa}\end{array}$ & $\begin{array}{c}Y_{[110]} \\
\mathrm{GPa}\end{array}$ & $\begin{array}{c}Y_{[111]} \\
\mathrm{GPa}\end{array}$ \\
\hline \hline $\mathrm{pSi}(\exp )$ & $134 \pm 5.3$ & $49 \pm 8.1$ & $62 \pm 1.2$ & $9.30 \pm 0.47$ & $-2.48 \pm 0.37$ & $16.21 \pm 0.37$ & $108 \pm 5.5$ & $134 \pm 2.7$ & $146 \pm 4.3$ \\
\hline bSi (Ref. $[26])$ & 165.7 & 63.9 & 79.6 & 7.68 & -2.14 & 12.6 & 130 & 169 & 188 \\
\hline
\end{tabular}

In the literature, one finds several recent studies trying to elucidate elastic properties of pSi by means of different experimental techniques. Aliev et al. [1] performed acoustic transmission spectroscopy to measure longitudinal sound velocities along the principal crystallographic directions in $\mathrm{pSi}$ as function of porosity $\Phi$ only. Their results, which show a characteristic $\Phi$-dependence of sound velocity, stiffness tensor $\sigma_{i j}$ and Young moduli however should be considered to be representative for the composite system of silicon and pores only. Not only average the used sound waves $(\lambda>1 \mu \mathrm{m})$ over many pores, their analysis crucially incorporates the macroscopic Gibson and Ashby model for Young moduli of cellular solids and does not provide microscopic properties of pSi. Grosman et al. [18] and Gor et al. [16] on the other hand measured adsorption induced strain in $\mathrm{pSi}$ with $R_{\text {Grosman }} \approx 6 \mathrm{~nm}$ and $R_{\text {Gor }} \approx 4 \mathrm{~nm}$. They used 
finite element calculations to relate macroscopic distortion of thin pSi plates to microscopic properties of pSi. Both studies agree on a sizable reduction of microscopic Young moduli in pSi maybe related to surface stress. The observation of reduced Young moduli agrees favorably with the results of our experiments. Based on a simplified, but theoretically manageable model of hexagonally arranged, cylindrical pores, Gor et al. state a Young modulus of $90 \mathrm{GPa}$ for the [001] direction in a morphologically similar pSi sample, compared to $108 \mathrm{GPa}$ reported in this study.

In the aftermath of these studies, the presented work is to the best of our knowledge the first model independent and comprehensive experimental determination of elastic properties of pSi. Our data analysis only resorted to the indisputable cubic structure of pSi when calculating $c_{i j}$ respectively $Y_{[H K L]}$. Furthermore it allowed extracting all three independent components of $c_{i j}$, which provides a full elastic characterization of the material, whereas preceding distortion studies $[18,16]$ appeared incomplete.

Here, one should also stress that the reported elastic constants (Table III) for pSi fulfill within the margin of error the Keating relation. It is $\Psi=0.95 \pm 0.05$. Our measurements constitute an independent confirmation of its validity in the structured system down to length scales of a few nanometers. Not only prevails the cubic structure in pSi. The tetrahedral coordination of Si atoms, as a prerequisite for the validity of the Keating relation, appears not significantly altered in the etched samples. Earlier studies [1] crucially incorporated the validity of Eqn 4 in their data analysis.

The question arises what underlying mechanism might be responsible for modified phonon dispersions in pSi. Native oxide layers on pore walls [15] are legitimate candidates to have an impact on the propagation of phonons in the nanostructured pSi membranes. Strain in pSi might enhance anharmonic contributions in the Si-Si interaction, but the bona-fide almost vanishing difference between bSi and pSi lattice constants seems to object such a scenario. Grosman et al. [18] stated surface stress as most likely cause for the reduced Young moduli in their samples. Another effect that might modify the dispersion relation in the Debye regime might be found in phonon confinement, which is also responsible for the formation of Lamb waves in nano-rods or thin nano-plates. Here, the boundary condition that the normal components of the stress tensor vanishes at interfaces drastically alters the allowed vibrational modes [33, 34, 2, 29]. The last scenario is at least in principle prone to theoretical studies. A proper calculation of the dispersion in pSi would however require a full quantum mechanical treatment due to the crystalline nature of the system. Such a demanding theoretical approach is beyond the scope of this experimental study.

Understanding the elastic constants and their origin in pSi respectively nanostructured silicon is naturally of interest for electronic devices that incorporate nanometer sized features and is expected to play an important role for envisioned sensor applications that rely on the elastic response of the device on external stimuli. The field of thermoelectrics also benefits from scrutinizing details of modified dispersions in nanostructured $\mathrm{Si}$, which is discussed as potential TEM of interest $[37,7,19,9,55,48,13,11,49,54]$. For instance, our 
experiments probed phonons that account for roughly $30 \%$ of the accumulated thermal conductivity $\kappa$ in $\mathrm{Si}$ at temperatures between $300 \mathrm{~K}$ and $1000 \mathrm{~K}$ [22]. Consequently, the evidently reduced sound velocities must have a sizable effect on scattering rates [32] and density of states. Both quantities enter critically $\kappa$ and by that the omnipresent figure of merit $Z T$.

\section{Conclusion}

Macroscopic amounts of single crystalline pSi with roughly $8 \mathrm{~nm}$ wide pores were synthesized by electrochemical etching for structural and dynamical studies on nanostructured silicon by means of elastic and inelastic scattering techniques. Elastic neutron diffraction experiments confirmed the cubic diamond structure of pSi. Voigt-shaped Bragg reflections were interpreted in terms of finite coherence lengths in the monolithic silicon membranes. From the Lorentz contribution to the profile coherence lengths of less than $60 \mathrm{~nm}$ but significantly larger than next nearest pore-pore distance were inferred.

Inelastic cold neutron scattering experiments allowed probing the phonon dispersion in the linear Debye regime for the high symmetry directions [001], [110], and [111]. Longitudinal and transverse modes were studied up to energy transfers of $4 \mathrm{meV}$. As a main result, the measurements found sound velocities reduced by roughly $10 \%$ compared to bSi. This reduction reflects significantly reduced elastic constants in the nanostructured material. For instance, the modified sound velocities translated themselves into smaller Young moduli, e.g. $108 \mathrm{GPa}=Y_{[111]}^{p S i}<Y_{[111]}^{b S i}=130 \mathrm{GPa}$. This observation agrees with recent studies on the elastic response of pSi upon water adsorption [16].

The fundamental origin of reduced sound velocities in pSi as evident from the modified dispersion is still under debate and a topic of active research. It might find an explanation in native oxide layers at the interfaces or surface stress. Modified boundary conditions for the stress tensor itself at the pore-silicon interface might also impact the dispersion relation [33, 29] and significantly alter the materials vibrational eigenmodes. But more experimental as well as theoretical studies appear necessary to achieve a deeper and more comprehensive understanding of the phenomena.

We demonstrated the potential of neutron scattering techniques to probe the phonon dispersion of nanostructured, crystalline materials, e.g., pSi in the Debye regime. It appears straightforward to perform such studies in the entire Brillouin zone by utilizing thermal neutron spectrometers. Employed techniques overcome the limitations of other techniques as Brillouin scattering and acoustic transmission spectroscopy, which only provide information close to the highsymmetry $\Gamma$-point. Consequently, our experimental approach is of particular interest in the field of nanophononics or thermoelectrics where phonon gap engineering or phonon dispersion engineering in general is expected to play an important role to improve or tailor device performances. Future challenges are not only to extract elastic properties of nanostructured Si-based systems but rather to access detailed information about atomistic interactions in nanostructures 
as encoded in the complex dispersion relation. With respect to thermoelectric research it is envisioned to scrutinize the subtle but sizable [57] influences of modified phonon dispersion relations on macroscopic quantities as the thermal conductivity and consequently the thermoelectric figure of merit $Z T$. These influences are usually ignored in theoretical studies not due to a lack of importance or even interest rather due to a lack of reliable data.

\section{Acknowledgment}

We thank Dr. Sebastian Risse (HZB) for access to the laser cutter. We thank Dr. Daniel Abou-Ras (HZB) for access to the SEM facility.

[1] Aliev, G. N., Goller, B., Snow, P. A., 2011. Elastic properties of porous silicon studied by acoustic transmission spectroscopy. Journal of Applied Physics 110 (4), 043534.

URL http://scitation.aip.org/content/aip/journal/jap/110/4/ $10.1063 / 1.3626790$

[2] Balandin, A., Wang, K. L., 07 1998. Significant decrease of the lattice thermal conductivity due to phonon confinement in a free-standing semiconductor quantum well. Physical Review B 58 (3), 1544-1549.

URL http://link.aps.org/doi/10.1103/PhysRevB.58.1544

[3] Balandin, A. A., 2005-07-01T00:00:00. Nanophononics: Phonon engineering in nanostructures and nanodevices. Journal of Nanoscience and Nanotechnology 5 (7), 1015-1022.

URL http://www.ingentaconnect.com/content/asp/jnn/2005/ 00000005/00000007/art00002

[4] Balzar, D., Oct 1992. Profile fitting of X-ray diffraction lines and Fourier analysis of broadening. Journal of Applied Crystallography 25 (5), 559-570. URL http://dx.doi .org/10.1107/S0021889892004084

[5] Barla, K., Herino, R., Bomchil, G., Pfister, J. C., Freund, A., 101984. Determination of lattice parameter and elastic properties of porous silicon by X-ray diffraction. Journal of Crystal Growth 68 (3), 727-732.

URL http://www.sciencedirect.com/science/article/pii/ 0022024884901118

[6] Barrett, E. P., Joyner, L. G., Halenda, P. P., 01 1951. The determination of pore volume and area distributions in porous substances. i. computations from nitrogen isotherms. Journal of the American Chemical Society 73 (1), 373-380.

URL http://dx.doi.org/10.1021/ja01145a126

[7] Boukai, A. I., Bunimovich, Y., Tahir-Kheli, J., Yu, J.-K., Goddard III, W. A., Heath, J. R., 01 2008. Silicon nanowires as efficient thermoelectric materials. Nature 451 (7175), 168-171.

URL http://dx.doi.org/10.1038/nature06458 
[8] Brunauer, S., Emmett, P. H., Teller, E., 02 1938. Adsorption of gases in multimolecular layers. Journal of the American Chemical Society 60 (2), 309-319.

URL http://dx.doi.org/10.1021/ja01269a023

[9] Bux, S. K., Blair, R. G., Gogna, P. K., Lee, H., Chen, G., Dresselhaus, M. S., Kaner, R. B., Fleurial, J.-P., 2009. Nanostructured bulk silicon as an effective thermoelectric material. Advanced Functional Materials 19 (15), $2445-2452$.

URL http://dx.doi.org/10.1002/adfm. 200900250

[10] Canham, L., 2014. Handbook of Porous Silicon. Handbook of Porous Silicon. Springer International Publishing.

URL https://books .google.de/books?id=pCrZoAEACAAJ

[11] Claudio, T., Schierning, G., Theissmann, R., Wiggers, H., Schober, H., Koza, M. M., Hermann, R. P., 2012. Effects of impurities on the lattice dynamics of nanocrystalline silicon for thermoelectric application. Journal of Materials Science 48 (7), 2836-2845.

URL http://dx.doi.org/10.1007/s10853-012-6827-y

[12] Coasne, B., Grosman, A., Ortega, C., Simon, M., 06 2002. Adsorption in noninterconnected pores open at one or at both ends: A reconsideration of the origin of the hysteresis phenomenon. Physical Review Letters 88 (25), $256102-$.

URL http://link.aps.org/doi/10.1103/PhysRevLett.88.256102

[13] de Boor, J., Kim, D. S., Ao, X., Becker, M., Hinsche, N. F., Mertig, I., Zahn, P., Schmidt, V., 2012. Thermoelectric properties of porous silicon. Applied Physics A 107 (4), 789-794.

URL http://dx.doi.org/10.1007/s00339-012-6879-5

[14] Efron, B., Tibshirani, R., 1994. An Introduction to the Bootstrap. Chapman \& Hall/CRC Monographs on Statistics \& Applied Probability. Taylor \& Francis.

[15] Fang, J., Pilon, L., 2012. Tuning thermal conductivity of nanoporous crystalline silicon by surface passivation: A molecular dynamics study. Applied Physics Letters 101 (1), 011909.

URL http://scitation.aip.org/content/aip/journal/apl/101/1/ $10.1063 / 1.4733352$

[16] Gor, G. Y., Bertinetti, L., Bernstein, N., Hofmann, T., Fratzl, P., Huber, P., 2015. Elastic response of mesoporous silicon to capillary pressures in the pores. Applied Physics Letters 106 (26), 261901.

URL http://scitation.aip.org/content/aip/journal/apl/106/26/ $10.1063 / 1.4923240$ 
[17] Goupil, C., Seifert, W., Zabrocki, K., Müller, E., Snyder, G. J., 2011. Thermodynamics of thermoelectric phenomena and applications. Entropy $13(8), 1481$.

URL http: //www.mdpi.com/1099-4300/13/8/1481

[18] Grosman, A., Puibasset, J., Rolley, E., 2015. Adsorption-induced strain of a nanoscale silicon honeycomb. EPL (Europhysics Letters) 109 (5), 56002. URL http://stacks. iop.org/0295-5075/109/i=5/a=56002

[19] Hao, Q., Chen, G., Jeng, M.-S., 2009. Frequency-dependent monte carlo simulations of phonon transport in two-dimensional porous silicon with aligned pores. Journal of Applied Physics 106 (11), 114321.

URL http://scitation.aip.org/content/aip/journal/jap/106/11/ $10.1063 / 1.3266169$

[20] Hao, Q., Xu, D., Lu, N., Zhao, H., 05 2016. High-throughput ZT predictions of nanoporous bulk materials as next-generation thermoelectric materials: A material genome approach. Physical Review B 93 (20), 205206-.

URL http://link.aps.org/doi/10.1103/PhysRevB.93.205206

[21] He, J., Amsler, M., Xia, Y., Naghavi, S. S., Hegde, V. I., Hao, S., Goedecker, S., Ozoliņš, V., Wolverton, C., 07 2016. Ultralow thermal conductivity in full heusler semiconductors. Physical Review Letters 117 (4), 046602-.

URL http://link.aps.org/doi/10.1103/PhysRevLett.117.046602

[22] Henry, A. S., Chen, G., 2008. Spectral phonon transport properties of silicon based on molecular dynamics simulations and lattice dynamics. Journal of Computational and Theoretical Nanoscience 5 (2), 141-152.

URL http://www.ingentaconnect.com/content/asp/jctn/2008/ 00000005/00000002/art00002

[23] Henschel, A., Hofmann, T., Huber, P., Knorr, K., 02 2007. Preferred orientations and stability of medium length $n$-alkanes solidified in mesoporous silicon. Physical Review E 75 (2), 021607-.

URL http://link.aps.org/doi/10.1103/PhysRevE.75.021607

[24] Hofmann, T., Dobisz, E., Ocko, B. M., 2009. Grazing incident small angle x-ray scattering: A metrology to probe nanopatterned surfaces. Journal of Vacuum Science \& Technology B 27 (6), 3238-3243.

URL http://scitation.aip.org/content/avs/journal/jvstb/27/6/ 10.1116/1.3253608

[25] Holmes, J. D., Ziegler, K. J., Doty, R. C., Pell, L. E., Johnston, K. P., Korgel, B. A., 04 2001. Highly luminescent silicon nanocrystals with discrete optical transitions. Journal of the American Chemical Society 123 (16), 3743-3748.

URL http://dx.doi.org/10.1021/ja002956f 
[26] Hopcroft, M., Nix, W., Kenny, T., April 2010. What is the young's modulus of silicon? Microelectromechanical Systems, Journal of 19 (2), 229-238.

[27] Hopkins, P. E., Phinney, L. M., Rakich, P. T., Olsson, R. H., El-Kady, I., 2010. Phonon considerations in the reduction of thermal conductivity in phononic crystals. Applied Physics A 103 (3), 575-579.

URL http://dx.doi .org/10.1007/s00339-010-6189-8

[28] Jauch, W., Reehuis, M., Jul 2005. Electron-density distribution in cubic $\mathrm{SrTiO}_{3}$ : a comparative $\gamma$-ray diffraction study. Acta Crystallographica Section A 61 (4), 411-417.

URL http://dx.doi.org/10.1107/S0108767305013231

[29] Kargar, F., Ramirez, S., Debnath, B., Malekpour, H., Lake, R. K., Balandin, A. A., 2015. Acoustic phonon spectrum and thermal transport in nanoporous alumina arrays. Applied Physics Letters 107 (17), 171904.

URL http://scitation.aip.org/content/aip/journal/apl/107/17/ $10.1063 / 1.4934883$

[30] Keating, P. N., 05 1966. Effect of invariance requirements on the elastic strain energy of crystals with application to the diamond structure. Physical Review 145 (2), 637-645.

URL http://link.aps.org/doi/10.1103/PhysRev.145.637

[31] Kim, W., 2015. Strategies for engineering phonon transport in thermoelectrics. J. Mater. Chem. C 3, 10336-10348.

URL http://dx.doi.org/10.1039/C5TC01670C

[32] Klemens, P. G., 1955. The scattering of low-frequency lattice waves by static imperfections. Proceedings of the Physical Society. Section A 68 (12), 1113.

URL http://stacks.iop.org/0370-1298/68/i=12/a=303

[33] Lamb, H., 03 1917. On waves in an elastic plate. Proceedings of the Royal Society of London A: Mathematical, Physical and Engineering Sciences 93 (648), 114-128.

URL http://rspa.royalsocietypublishing.org/content/93/648/ 114. abstract

[34] Landau, L. D., Lifshits, E. M., 1986. Theory of Elasticity. ButterworthHeinemann.

URL https://books.google.de/books?id=tpY-VkwCkAIC

[35] Landers, J., Gor, G. Y., Neimark, A. V., 11 2013. Density functional theory methods for characterization of porous materials. Colloids and Surfaces A: Physicochemical and Engineering Aspects 437, 3-32.

URL http://www.sciencedirect.com/science/article/pii/ S0927775713000277 
[36] Le, M. D., Quintero-Castro, D. L., Toft-Petersen, R., Groitl, F., Skoulatos, M., Rule, K. C., Habicht, K., 11 2013. Gains from the upgrade of the cold neutron triple-axis spectrometer FLEXX at the BER-II reactor. Nuclear Instruments and Methods in Physics Research Section A: Accelerators, Spectrometers, Detectors and Associated Equipment 729, 220-226.

URL http://www.sciencedirect.com/science/article/pii/ S0168900213009832

[37] Lee, J.-H., Galli, G. A., Grossman, J. C., 2008. Nanoporous si as an efficient thermoelectric material. Nano Letters 8 (11), 3750-3754.

URL http://dx.doi.org/10.1021/nl802045f

[38] Lemal, S., Nguyen, N., de Boor, J., Ghosez, P., Varignon, J., Klobes, B., Hermann, R. P., Verstraete, M. J., 11 2015. Thermoelectric properties of the unfilled skutterudite $\mathrm{FeSb}_{3}$ from first principles and seebeck local probes. Physical Review B 92 (20), 205204-.

URL http://link.aps.org/doi/10.1103/PhysRevB.92.205204

[39] Lim, J., Wang, H.-T., Tang, J., Andrews, S. C., So, H., Lee, J., Lee, D. H., Russell, T. P., Yang, P., 01 2016. Simultaneous thermoelectric property measurement and incoherent phonon transport in holey silicon. ACS Nano 10 (1), 124-132.

URL http://dx.doi.org/10.1021/acsnano.5b05385

[40] Lorensen, W. E., Cline, H. E., 1987. Marching cubes: A high resolution 3D surface construction algorithm. In: Proceedings of the 14th Annual Conference on Computer Graphics and Interactive Techniques. SIGGRAPH '87. ACM, New York, NY, USA, pp. 163-169.

URL http://doi.acm.org/10.1145/37401.37422

[41] Magnuson, M., Mattesini, M., Nong, N. V., Eklund, P., Hultman, L., 05 2012. Electronic-structure origin of the anisotropic thermopower of nanolaminated ti $\$\{\}$ _ $\{3\} \$$ sic $\$\{\}\{\{2\} \$$ determined by polarized x-ray spectroscopy and seebeck measurements. Physical Review B 85 (19), 195134-. URL http://link.aps.org/doi/10.1103/PhysRevB.85.195134

[42] Mayer, H., Knorr, K., Többens, D., Stüßer, N., Lampert, G., 102001. E9: The new high-resolution neutron powder diffractometer at the Berlin neutron scattering center. In: European Powder Diffraction EPDIC 7. Vol. 378 of Materials Science Forum. Trans Tech Publications, pp. 288-293.

[43] Melosh, N. A., Boukai, A., Diana, F., Gerardot, B., Badolato, A., Petroff, P. M., Heath, J. R., 04 2003. Ultrahigh-density nanowire lattices and circuits. Science 300 (5616), 112-115.

URL http://science.sciencemag.org/content/300/5616/112. abstract

[44] Mu, X., Wang, L., Yang, X., Zhang, P., To, A. C., Luo, T., 112015. Ultra-low thermal conductivity in si/ge hierarchical superlattice nanowire. 
Scientific Reports 5, 16697 EP -.

URL http://dx.doi.org/10.1038/srep16697

[45] Mukherjee, S., Givan, U., Senz, S., Bergeron, A., Francoeur, S., de la Mata, M., Arbiol, J., Sekiguchi, T., Itoh, K. M., Isheim, D., Seidman, D. N., Moutanabbir, O., 06 2015. Phonon engineering in isotopically disordered silicon nanowires. Nano Letters 15 (6), 3885-3893.

URL http://dx.doi.org/10.1021/acs.nanolett.5b00708

[46] Page, A., Uher, C., Poudeu, P. F., Van der Ven, A., 11 2015. Phase separation of full-heusler nanostructures in half-heusler thermoelectrics and vibrational properties from first-principles calculations. Physical Review B $92(17), 174102-$.

URL http://link.aps.org/doi/10.1103/PhysRevB.92.174102

[47] Qu, Y., Liao, L., Li, Y., Zhang, H., Huang, Y., Duan, X., 12 2009. Electrically conductive and optically active porous silicon nanowires. Nano Letters 9 (12), 4539-4543.

URL http://dx.doi.org/10.1021/nl903030h

[48] Romano, G., Di Carlo, A., Grossman, J., 2012. Mesoscale modeling of phononic thermal conductivity of porous si: interplay between porosity, morphology and surface roughness. Journal of Computational Electronics 11 (1), 8-13.

URL http://dx.doi.org/10.1007/s10825-012-0390-2

[49] Romano, G., Grossman, J. C., 2014. Toward phonon-boundary engineering in nanoporous materials. Applied Physics Letters 105 (3), 033116.

URL http://scitation.aip.org/content/aip/journal/apl/105/3/ $10.1063 / 1.4891362$

[50] Sailor, M., 2012. Porous Silicon in Practice: Preparation, Characterization and Applications. Wiley.

URL https://books.google.de/books?id=QIjMWp41GMsC

[51] Shirane, G., Shapiro, S., Tranquada, J., 2002. Neutron Scattering with a Triple-Axis Spectrometer: Basic Techniques. Cambridge University Press. URL https://books.google.de/books?id=agc8GeLZ-joC

[52] Snyder, G. J., Toberer, E. S., 02 2008. Complex thermoelectric materials. Nature Publishing Group 7 (2), 105-114.

URL http://dx.doi.org/10.1038/nmat2090

[53] Suzuki, T., Kikkawa, A., Tokura, Y., Taguchi, Y., 04 2016. Impact of electron doping on thermoelectric properties in filled skutterudite $\$\{\backslash$ mathrm $\{$ irsb $\}\}-\{3\} \$$. Physical Review B 93 (15), 155101-.

URL http://link.aps.org/doi/10.1103/PhysRevB.93.155101 
[54] Tang, G. H., Bi, C., Fu, B., 2013. Thermal conduction in nano-porous silicon thin film. Journal of Applied Physics 114 (18), 184302.

URL http://scitation.aip.org/content/aip/journal/jap/114/18/ $10.1063 / 1.4829913$

[55] Tang, J., Wang, H.-T., Lee, D. H., Fardy, M., Huo, Z., Russell, T. P., Yang, P., 10 2010. Holey silicon as an efficient thermoelectric material. Nano Letters 10 (10), 4279-4283.

URL http://dx.doi.org/10.1021/nl102931z

[56] Uher, C., Yang, J., Hu, S., Morelli, D. T., Meisner, G. P., 04 1999. Transport properties of pure and doped $\backslash \operatorname{textit}\{\mathrm{m}\}$ nisn $(\backslash \operatorname{textit}\{\mathrm{m}\}=\mathrm{zr}, \mathrm{hf}$ ). Physical Review B 59 (13), 8615-8621.

URL http://link.aps.org/doi/10.1103/PhysRevB.59.8615

[57] Wingert, M. C., Kwon, S., Hu, M., Poulikakos, D., Xiang, J., Chen, R., 04 2015. Sub-amorphous thermal conductivity in ultrathin crystalline silicon nanotubes. Nano Letters 15 (4), 2605-2611.

URL http://dx.doi.org/10.1021/acs.nanolett.5b00167

[58] Yang, K., Cahangirov, S., Cantarero, A., Rubio, A., D’Agosta, R., 03 2014. Thermoelectric properties of atomically thin silicene and germanene nanostructures. Physical Review B 89 (12), 125403-.

URL http://link.aps.org/doi/10.1103/PhysRevB.89.125403

[59] Zhang, X. G., 01 2004. Morphology and formation mechanisms of porous silicon. Journal of The Electrochemical Society 151 (1), C69-C80.

URL http://jes.ecsdl .org/content/151/1/C69

[60] Zhu, T., Fu, C., Xie, H., Liu, Y., Zhao, X., 2015. High efficiency half-heusler thermoelectric materials for energy harvesting. Advanced Energy Materials 5 (19), 1500588.

URL http://dx.doi.org/10.1002/aenm. 201500588 